\title{
HIF1A (rs1 1549465) and AKNA (rs10817595) Gene Polymorphisms Are Associated with Primary Sjögren's Syndrome
}

\author{
Gabriela Hernández-Molina, ${ }^{1}$ José Manuel Rodríguez-Pérez, ${ }^{2}$ \\ Javier Fernández-Torres, ${ }^{3,4}$ Guadalupe Lima, ${ }^{1}$ Nonanzit Pérez-Hernández, ${ }^{2}$ \\ Alberto López-Reyes, ${ }^{3}$ and Gabriela Angélica Martínez-Nava ${ }^{3}$ \\ ${ }^{1}$ Department of Immunology and Rheumatology, Instituto Nacional de Ciencias Médicas y Nutrición Salvador Zubirán, \\ Mexico City, Mexico \\ ${ }^{2}$ Department of Molecular Biology, Instituto Nacional de Cardiología Ignacio Chávez, Mexico City, Mexico \\ ${ }^{3}$ Synovial Fluid Laboratory, Instituto Nacional de Rehabilitación Luis Guillermo Ibarra Ibarra, Mexico City, Mexico \\ ${ }^{4}$ Biological and Health Sciences PhD Program, Universidad Autónoma Metropolitana Iztapalapa, Mexico City, Mexico
}

Correspondence should be addressed to Gabriela Angélica Martínez-Nava; ameria.justice@gmail.com

Received 23 January 2017; Revised 6 March 2017; Accepted 23 March 2017; Published 6 April 2017

Academic Editor: Louise E. Glover

Copyright (c) 2017 Gabriela Hernández-Molina et al. This is an open access article distributed under the Creative Commons Attribution License, which permits unrestricted use, distribution, and reproduction in any medium, provided the original work is properly cited.

\begin{abstract}
Objective. To evaluate the allele and genotype frequencies of polymorphic sites of HIF1A and ANKA genes in primary Sjögren's syndrome (pSS). Methods. We included 110 patients with pSS and 141 ethnically matched healthy controls. Three HIF1A gene polymorphisms (Pro582Ser, Ala588Thr, and C191T) and two AKNA gene polymorphisms ( $1372 \mathrm{C}>$ A and Pro624Leu) were genotyped using TaqMan probes in a Real-Time PCR instrument. Associations between pSS and genotypes, alleles, and inheritance models of the SNPs of interest were evaluated by logistic regression adjusted by age and gender. Results. The C/T genotype and the $T$ allele of the HIF1A Pro582Ser polymorphism protected against pSS $(\mathrm{OR}=0.22 ; 95 \% \mathrm{CI}=0.09-0.52 ; P<0.01 ; \mathrm{OR}=0.26 ; 95 \%$ $\mathrm{CI}=0.12-0.58 ; P<0.01$, resp.), whereas under a recessive model adjusted by age and gender, the $A K N A-1372 C>A$ polymorphism $\mathrm{A} / \mathrm{A}$ genotype was associated with an increased risk of pSS $(\mathrm{OR}=2.60 ; 95 \% \mathrm{CI}=1.11-6.12 ; P=0.03)$. Conclusions. We identified HIF1A Pro582Ser T allele and C/T genotype as well as $A K N A-1372 C>A$ polymorphism A/A genotype as genetic factors associated with pSS. Further studies in other populations are needed to validate our findings and research is warranted in order to shed some light on their functional implications across biological pathways in this disease.
\end{abstract}

\section{Introduction}

Primary Sjögren's syndrome (pSS) is a chronic autoimmune disorder characterized by diminished lacrimal and salivary gland secretion, resulting in keratoconjunctivitis sicca and xerostomia [1]. Patients with pSS mount a localized immunological response against the epithelial component of exocrine glands which produces a destructive inflammatory infiltrate comprised of activated $\mathrm{T}$ and $\mathrm{B}$ cells, which ultimately lead to a progressive exocrine function lost [2]. Epidemiological and genetic studies support the idea of a complex etiology of the disease, involving variants in numerous genes with functional implications across multiple biological pathways, including inflammation and oxidative burst $[3,4]$.

In these pathways, one of the key molecules is the hypoxia inducible factor- $1 \alpha$ (HIF- $1 \alpha$ ), which is the main transcriptional regulator of cellular and developmental response to hypoxia. The deregulation and overexpression as well as genetics variants of this gene have been related to angiogenesis, cell proliferation, metabolism, cancer biology, and osteoarthritis $[5,6]$. Although the importance of HIF- $1 \alpha$ is well described in the context of hypoxia, recent data suggest 
that it may also play a role in innate immunity. HIF-1 $\alpha$ is also a key player in the integration of the $\mathrm{T}$ cell receptor (TCR) and cytokine receptor-mediated signals of CD4+ helper $\mathrm{T}$ cells [7]. In addition, HIF-1 $\alpha$ enhances Th17 development through direct transcriptional activation of RAR-related orphan receptor gamma $\mathrm{t}(\mathrm{ROR} \gamma \mathrm{t})$ [8]. This subpopulation is highly increased in salivary gland tissue of patients with Sjögren's syndrome (SS) and in a mouse model of SS [9]. Conversely, in a HIF- $1 \alpha$ conditional knockout mouse model with dry-eye, the lacrimal glands exhibited increased acinar cell apoptosis and loss of glandular structures and polarities and, on the other hand, the blockage of HIF-1 $\alpha$ COX-2 axis enhanced inflammation [10]. Furthermore, the participation of HIF- $1 \alpha$ has been studied in inflammatory and autoimmune disorders. There is evidence that points out the key role of HIF- $1 \alpha$ in the regulation of several pathophysiological processes such as rheumatoid arthritis (RA) and systemic lupus erythematous, especially producing synovial inflammation as well as glomerular and interstitial inflammation [11, 12].

Another transcription factor involved in the immune response is the AT-hook transcription factor (AKNA). This protein targets genes with A/T-rich promoters and regulates specific genes expression involved in the establishment of the immune response. Particularly, AKNA upregulates the expression of two costimulatory molecules, CD40 and CD40L, in T and B-lymphocytes, as well as natural killer and dendritic cells [13]. To date, AKNA gene polymorphisms as well as its expression had been associated with other human pathologies in which the immune system plays an important role such as cervical cancer [14], leukemia [15], VogtKoyanagi-Harada's syndrome [16], and hepatitis C virus infection [17].

The aforementioned evidence suggests the involvement of HIF1A and AKNA genes in immune system pathologies. In this context, identifying genetic variants of these two genes in pSS will allow a more precise definition of pathogenic mechanisms of the disease.

The aim of this study was to explore the role of HIF1A and $A K N A$ gene polymorphisms and their association with pSS in a well characterized-clinical cohort of Mexican patients. We also analyzed the association of glandular, extraglandular, and serological features among pSS patients with these genes polymorphisms that codify transcriptional regulator factors expressed in $\mathrm{T}$ and $\mathrm{B}$ cells.

\section{Materials and Methods}

2.1. Subjects. We included 110 consecutive Mexican mestizo patients with the diagnosis of pSS according to the American-European Consensus Group SS classification criteria [18], who regularly attended a tertiary referral care center at Mexico City. Patient's clinical records were carefully reviewed according to a preestablished protocol. We registered demographic, glandular features (oral and ocular symptoms, parotid enlargement, Schirmer-I test, nonstimulated whole salivary flow (NSWSF), and fluorescein staining) and extraglandular features (nonerosive arthritis, neurological involvement [polyneuropathy, mononeuropathy, cranial nerves involvement, dysmyelinization, disautonomy radiographic or electrophysiology diagnosed, or biopsy proven], skin vasculitis [palpable purpura or biopsy proven], and Raynaud's phenomenon), as well as serological variables (anti-Ro/SSA and anti-La/SSB antibodies). We excluded patients with another concomitant autoimmune disease.

We included 141 healthy volunteers from a referral orthopedic center at the same city where the patients were recruited. Although they did not have complete evaluation to rule out the presence of pSS, they did not complain of oral/ocular symptoms and did not self-report any autoimmune condition.

All participants were not related and self-reported Mexican mestizo ancestry (at least three generations including their own).

2.2. Genetic Analysis. Genomic DNA was extracted from leucocytes, after collecting a peripheral blood sample using the Lahiri and Numberger method [19]. We evaluate three codifying polymorphisms of HIF1A gene, two missense (Pro582Ser, rs11549465 and Ala588Thr, rs11549467) and one $3^{\prime}$ UTR (C191T, rs2057482) and two AKNA gene polymorphisms, one in the promoter region $(-1372 C>A, \mathrm{rs} 10817595)$ and other in the coding region (Pro624Leu, rs3748176).

The SNPs were genotyped using $5^{\prime}$ exonuclease TaqMan genotyping assays on a StepOne Plus Real-Time PCR system (Applied Biosystems, Foster City, USA). The results were analyzed in allelic discrimination software StepOnePlus v2.3. Previously sequenced samples of the five polymorphisms of all different genotypes were included as positive controls and the results were found to be consistent.

2.3. Statistical Analysis. Hardy-Weinberg equilibrium (HWE) was calculated for each polymorphism using Fisher's exact test. According to the variable type, patients and controls were compared using Mann-Whitney $U$ test or Fisher's exact test when appropriate. Allelic and genotypic frequencies were estimated for all polymorphisms (rs11549465, rs11549467, and rs2057482 of HIF1A gene; rs10817595 and rs3748176 of AKNA gene) and inheritance models were constructed and assessed for the polymorphisms whose frequencies allowed it.

The associations between pSS, genotypes, alleles, and inheritance models of the SNPs of interest were evaluated by logistic regression models adjusted by age and gender. In order to reject a possible type I error, bootstrap-resampling analysis (100 replications) was performed. All the statistical analyses and graphs were performed using the statistical package STATA v12.1. (StataCorp, Texas, USA) and considering a significance level with $\alpha=0.05$.

2.4. Ethical Considerations. The study complies with the Declaration of Helsinki and was approved by the Institutional Bioethics and Research Committee of the Instituto Nacional de Ciencias Médicas y Nutrición. All participants signed an informed consent letter.

\section{Results}

3.1. Characteristics of the Study Population. Most of the patients were females (95.4\%), with a median age of 55 years and median disease duration of 7.8 years. Table 1 depicts their 
TABLE 1: Clinical and serological characteristics of PSS patients.

\begin{tabular}{lc}
\hline Variable & Primary Sjögren's syndrome $(n=110)$ \\
\hline Median age in years (interquartile range) & $55(17)$ \\
Female, $n$ (\%) & $105(95.45)$ \\
Median disease duration in years (interquartile range) & $7.8(10)$ \\
Ocular symptoms, $n$ (\%) & $90(90.9)$ \\
Oral symptoms, $n$ (\%) & $103(93.6)$ \\
Parotid enlargement, $n(\%)$ & $60(54.6)$ \\
Positive Schirmer-I test, $n(\%)$ & $96(87.3)$ \\
Impaired nonstimulated whole salivary flow, $n(\%)$ & $96 / 103(93.2)$ \\
Positive fluorescein staining, $n(\%)$ & $53 / 80(66.2)$ \\
Positive anti-Ro/SSA antibodies, $n(\%)$ & $91(82.7)$ \\
Positive anti-La/SSB antibodies, $n$ (\%) & $53(48.18)$ \\
Skin vasculitis, $n$ (\%) & $2(1.8)$ \\
Neurological involvement, $n(\%)$ & $12(10.9)$ \\
Raynaud's phenomenon $n(\%)$ & $11(10)$ \\
Nonerosive arthritis, $n(\%)$ & $23(20.9)$
\end{tabular}

clinical and serological features. In contrast, in the control group most of the subjects were males $(30.8 \%$ versus $4.5 \%$ pSS, $P<0.01$ ) and younger than the pSS group (41 versus 55 y/o, resp., $P<0.01)$.

3.2. Allele and Genotype Frequencies. Allele and genotype frequencies of polymorphic sites in patients with pSS and controls are depicted in Table 2. Observed and expected frequencies of all polymorphisms were in HWE in the total sample size. The genotypic frequencies of HIF1A Pro582Ser (rs11549465) and $A K N A-1372 C>A$ (rs10817595) polymorphisms were not in HWE in the control group $(P=$ 0.04 and $P=0.01$, resp.); nevertheless after Bonferroni correction for multiple comparisons the observed and expected genotypic frequencies given HWE for both polymorphisms were not statistically different in this group (Bonferroni corrected $\alpha=0.003$ ).

A similar distribution of HIF1A (rs11549467 and rs207482) as well as $A K N A$ (rs3748176) polymorphisms was observed. In contrast, a lower frequency of the $C / T$ genotype and the $T$ allele of the HIF1A (rs11549465) polymorphism was found among patients with pSS. In addition in the inheritance model, we found a higher frequency of the $A / A$ genotype of the AKNA (rs10817595) gene polymorphism in pSS (Table 2).

In the logistic regression adjusted by age and gender, the $C / T$ genotype and the $T$ allele of the HIF1A (rs11549465) polymorphism protected against pSS $(\mathrm{OR}=0.22 ; 95 \% \mathrm{CI}=$ $0.09-0.52 ; P<0.01 ; \mathrm{OR}=0.26 ; 95 \% \mathrm{CI}=0.12-0.58 ; P<0.01$, resp.) (Table 3 ).

On the other hand, under a recessive inheritance model adjusted by age and gender, the AKNA (rs10817595) $A / A$ genotype was associated with increased risk of pSS $(\mathrm{OR}=$ 2.60; 95\% CI $=1.11-6.12 ; P=0.03$ ) (Table 3).

We explored the association of glandular, extraglandular, and serological features among pSS patients and the associated HIF1A and AKNA gene polymorphisms (data not shown). Parotid enlargement was the only feature that was associated with the HIF1A gene polymorphism (rs11549465), conferring protection for the $C / T$ genotype versus $C / C$ (OR $=0.22 ; 95 \%$ CI $0.05-0.90 ; P=0.03)$. In addition, the allele $T$ of the same polymorphism (rs11549465) also conferred protection when compared with the $C$ allele $(\mathrm{OR}=0.24$; 95\% CI $=0.65-0.95 ; P=0.04)$. We did not find any other association with the rest of the HIF1A or AKNA gene polymorphisms tested.

\section{Discussion}

The pathogenesis of Sjögren's syndrome likely involves complex interactions between genes and environment; thus the study of genetic factors is an important area of research. For instance, genetic variation may influence molecular processes such as splicing and posttranslational modifications [3]. Herein, we report the allele and genotype frequencies of polymorphic sites of HIF1A and ANKA genes in pSS patients and their association with the susceptibility to this disease.

HIF- $1 \alpha$ regulates the expression of over 100 genes that control the major cellular functions including apoptosis, cell proliferation, glucose metabolism, erythropoiesis, iron metabolism, and angiogenesis. It is a master regulator of the cellular response to hypoxia and is widely expressed in all immune system cells. HIF1A expression can be triggered not only by hypoxia, but also by diverse stimuli associated with leukocyte activation and inflammation $[20,21]$. In this sense, several inflammatory cytokines, such as IL-1, TNF- $\alpha$, IL-4, and TGF- $\beta$, have been shown to induce HIF- $1 \alpha$ in multiple cell types during normoxic conditions. Induced HIF- $1 \alpha$ may specifically play a role in the innate immune response to viral infection; however it is still unknown if there is a relationship with type I IFN (IFN- $\alpha$ or IFN- $\beta$ ) and type II IFN (IFN- $\gamma$ ) [22].

On the other hand, there is evidence showing that HIF$1 \alpha$ upregulates the expression of inflammatory cytokines including IFN- $\gamma$ in synovial fibroblast of RA patients [23]. In this sense, IFN $\gamma$ has been associated with more severe forms 
TABLE 2: Genotype and allele frequency by study group of HIF1A and AKNA polymorphisms.

\begin{tabular}{|c|c|c|c|c|}
\hline \multirow{2}{*}{ Polymorphism } & \multicolumn{3}{|c|}{ Frequency $n(\%)$} & \multirow{2}{*}{$P$ value } \\
\hline & Total study population $(n=251)$ & Controls $(n=141)$ & Primary Sjögren's syndrome $(n=110)$ & \\
\hline \multicolumn{5}{|c|}{ HIF1A Pro582Ser (rs11549465) } \\
\hline $\mathrm{C} / \mathrm{C}$ & $188(78.0)$ & $94(69.6)$ & $94(88.7)$ & \multirow[t]{3}{*}{$<0.01$} \\
\hline $\mathrm{C} / \mathrm{T}$ & $53(22.0)$ & $41(30.4)$ & $12(11.3)$ & \\
\hline $\mathrm{T} / \mathrm{T}$ & 0 & 0 & 0 & \\
\hline \multicolumn{5}{|l|}{ Alleles } \\
\hline $\mathrm{C}$ & $429(89.0)$ & $229(84.8)$ & $200(94.3)$ & \multirow[t]{3}{*}{$<0.01$} \\
\hline $\mathrm{T}$ & $53(11.0)$ & $41(15.2)$ & $12(5.70)$ & \\
\hline $\mathrm{HWE}^{\mathrm{a}} P$ value & 0.09 & 0.04 & 0.99 & \\
\hline \multicolumn{5}{|c|}{ HIF1A Ala588Thr (rs11549467) } \\
\hline $\mathrm{G} / \mathrm{G}$ & $198(99.5)$ & $90(98.9)$ & $108(100.0)$ & \multirow[t]{3}{*}{0.46} \\
\hline $\mathrm{G} / \mathrm{A}$ & $1(0.5)$ & $1(1.10)$ & 0 & \\
\hline $\mathrm{A} / \mathrm{A}$ & 0 & 0 & 0 & \\
\hline \multicolumn{5}{|l|}{ Alleles } \\
\hline G & $397(99.8)$ & $181(99.4)$ & $216(100.0)$ & \multirow[t]{3}{*}{0.46} \\
\hline A & $1(0.25)$ & $1(0.60)$ & 0 & \\
\hline HWE $P$ value & 0.99 & 0.99 & - & \\
\hline \multicolumn{5}{|c|}{ HIF1A C191T (rs2057482) } \\
\hline $\mathrm{T} / \mathrm{T}$ & $190(83.0)$ & $96(80.7)$ & $94(85.4)$ & \multirow[t]{3}{*}{0.38} \\
\hline $\mathrm{T} / \mathrm{C}$ & $39(17.0)$ & $23(19.3)$ & $16(14.6)$ & \\
\hline $\mathrm{C} / \mathrm{C}$ & 0 & 0 & 0 & \\
\hline \multicolumn{5}{|l|}{ Alleles } \\
\hline $\mathrm{T}$ & $419(91.5)$ & $215(90.3)$ & $204(92.7)$ & \multirow[t]{3}{*}{0.40} \\
\hline $\mathrm{C}$ & $39(8.52)$ & $23(9.7)$ & $16(7.27)$ & \\
\hline HWE $P$ value & 0.38 & 0.60 & 0.99 & \\
\hline \multicolumn{5}{|c|}{$A K N A-1372 C>A(r s 10817595)$} \\
\hline $\mathrm{C} / \mathrm{C}$ & $72(29.2)$ & $41(29.1)$ & $31(29.2)$ & \multirow[t]{3}{*}{0.11} \\
\hline $\mathrm{C} / \mathrm{A}$ & $137(55.5)$ & $84(59.6)$ & $53(50.0)$ & \\
\hline $\mathrm{A} / \mathrm{A}$ & $38(15.4)$ & $16(11.3)$ & $22(20.8)$ & \\
\hline \multicolumn{5}{|c|}{ Inheritance models } \\
\hline $\mathrm{C} / \mathrm{A}+\mathrm{A} / \mathrm{A}$ & $175(70.85)$ & $100(70.9)$ & $75(70.8)$ & 0.54 \\
\hline $\mathrm{A} / \mathrm{A}^{\mathrm{b}}$ & $38(15.4)$ & $16(11.3)$ & $22(20.8)$ & 0.03 \\
\hline \multicolumn{5}{|l|}{ Alleles } \\
\hline $\mathrm{C}$ & $281(56.9)$ & $166(58.9)$ & $115(54.2)$ & \multirow[t]{3}{*}{0.18} \\
\hline A & $213(43.1)$ & $116(41.1)$ & $97(45.8)$ & \\
\hline HWE $P$ value & 0.05 & 0.01 & 0.99 & \\
\hline \multicolumn{5}{|c|}{ AKNA Pro624Leu (rs3748176) } \\
\hline $\mathrm{G} / \mathrm{G}$ & $69(28.1)$ & $39(28.1)$ & $30(28.3)$ & \multirow[t]{3}{*}{0.56} \\
\hline G/A & $132(53.9)$ & $78(56.1)$ & $54(50.9)$ & \\
\hline $\mathrm{A} / \mathrm{A}$ & $44(18.0)$ & $22(20.8)$ & $22(20.8)$ & \\
\hline \multicolumn{5}{|c|}{ Inheritance models } \\
\hline $\mathrm{G} / \mathrm{A}+\mathrm{A} / \mathrm{A}$ & $176(71.8)$ & $100(71.9)$ & $76(71.7)$ & 0.54 \\
\hline $\mathrm{A} / \mathrm{A}^{\mathrm{b}}$ & $44(18.0)$ & $22(20.8)$ & $22(20.8)$ & 0.20 \\
\hline Alleles & & & & \\
\hline G & $270(55.1)$ & $156(56.1)$ & $114(53.8)$ & 0.34 \\
\hline $\mathrm{A}$ & $220(44.9)$ & $122(43.9)$ & $98(46.2)$ & \\
\hline HWE $P$ value & 0.20 & 0.12 & 0.85 & \\
\hline
\end{tabular}

${ }^{\mathrm{a}} \mathrm{HWE}$ : Hardy Weinberg Equilibrium.

${ }^{\mathrm{b}}$ Recessive inheritance model; the reference group is formed by C/A and C/C genotype carriers or G/A and G/G genotype carriers for each AKNA polymorphism, respectively.

${ }^{c}$ Fisher's exact test $P$ value. 
TABLE 3: Odds ratio for primary Sjögren's syndrome by polymorphisms genotypes, alleles, and inheritance models.

\begin{tabular}{|c|c|c|c|}
\hline Polymorphism & $N$ (controls/cases) & $\mathrm{OR}^{\mathrm{a}}(95 \% \mathrm{CI})$ & $P$ value \\
\hline \multicolumn{4}{|c|}{ HIF1A Pro582Ser (rs11549465) } \\
\hline $\mathrm{C} / \mathrm{C}$ & $94 / 94$ & 1.00 & \\
\hline $\mathrm{C} / \mathrm{T}$ & $41 / 12$ & $0.22(0.09-0.52)^{\mathrm{c}}$ & $<0.01$ \\
\hline $\mathrm{T} / \mathrm{T}$ & $0 / 0$ & - & - \\
\hline \multicolumn{4}{|l|}{ Alleles } \\
\hline $\mathrm{C}$ & $229 / 200$ & 1.00 & \\
\hline $\mathrm{T}$ & $41 / 12$ & $0.26(0.12-0.58)^{\mathrm{c}}$ & $<0.01$ \\
\hline \multicolumn{4}{|c|}{ HIF1A C191T (rs2057482) } \\
\hline $\mathrm{T} / \mathrm{T}$ & $96 / 94$ & 1.00 & \\
\hline $\mathrm{T} / \mathrm{C}$ & $23 / 16$ & $0.43(0.18-1.04)$ & 0.06 \\
\hline $\mathrm{C} / \mathrm{C}$ & $0 / 0$ & - & - \\
\hline \multicolumn{4}{|l|}{ Alleles } \\
\hline $\mathrm{T}$ & $215 / 204$ & 1.00 & \\
\hline $\mathrm{C}$ & $23 / 16$ & $0.47(0.21-1.07)$ & 0.07 \\
\hline \multicolumn{4}{|c|}{$A K N A-1372 C>A(r s 10817595)$} \\
\hline $\mathrm{C} / \mathrm{C}$ & $41 / 31$ & 1.00 & \\
\hline $\mathrm{C} / \mathrm{A}$ & $84 / 53$ & $0.64(0.31-1.32)$ & 0.23 \\
\hline $\mathrm{A} / \mathrm{A}$ & $16 / 22$ & $1.94(0.73-5.15)$ & 0.19 \\
\hline \multicolumn{4}{|c|}{ Inheritance models } \\
\hline $\mathrm{C} / \mathrm{A}+\mathrm{A} / \mathrm{A}$ & $100 / 75$ & $0.82(0.41-1.64)$ & 0.58 \\
\hline $\mathrm{A} / \mathrm{A}^{\mathrm{b}}$ & $16 / 22$ & $2.60(1.11-6.12)^{\mathrm{c}}$ & 0.03 \\
\hline \multicolumn{4}{|l|}{ Alleles } \\
\hline $\mathrm{C}$ & $166 / 115$ & 1.00 & \\
\hline $\mathrm{A}$ & $116 / 97$ & $1.20(0.78-1.86)$ & 0.42 \\
\hline \multicolumn{4}{|c|}{ AKNA Pro624Leu (rs3748176) } \\
\hline $\mathrm{G} / \mathrm{G}$ & $39 / 30$ & 1.00 & \\
\hline G/A & $78 / 54$ & $0.76(0.37-1.58)$ & 0.46 \\
\hline $\mathrm{A} / \mathrm{A}$ & $22 / 22$ & $1.56(0.62-3.96)$ & 0.35 \\
\hline \multicolumn{4}{|c|}{ Inheritance models } \\
\hline $\mathrm{G} / \mathrm{A}+\mathrm{A} / \mathrm{A}$ & $100 / 76$ & $0.92(0.46-1.83)$ & 0.80 \\
\hline $\mathrm{A} / \mathrm{A}^{\mathrm{b}}$ & $22 / 22$ & $1.88(0.85-4.16)$ & 0.12 \\
\hline \multicolumn{4}{|l|}{ Alleles } \\
\hline G & $156 / 114$ & 1.00 & \\
\hline A & $122 / 98$ & $1.17(0.75-1.81)$ & 0.48 \\
\hline
\end{tabular}

${ }^{\text {a }}$ Odds ratio adjusted by age and gender.

${ }^{\mathrm{b}}$ Recessive inheritance model; the reference group is formed by C/A and C/C genotype carriers or G/A and G/G genotype carriers for each AKNA polymorphism, respectively.

${ }^{\mathrm{c}}$ Odds ratio statistically significant after bootstrap resampling (100 repetitions).

of SS, characterized by high grade infiltration of lymphocytes, macrophages, and dendritic cells as well as with gastric NHL lymphomas [24].

HIF- $1 \alpha$ also participates in the integration of the TCR and cytokine receptor-mediated signals of $\mathrm{CD} 4+$ helper lineage (e.g., the activation of P13 kinase/mTOR pathway) as well as in $\mathrm{CD} 8+$ effectors differentiation, in a fashion independent of oxygen availability [7]. HIF-1 $\alpha$ enhances Th17 development and also attenuates Treg development by binding to transcription factor Foxp3 and targeting it for proteasomal degradation [8], although paradoxically it is also required for optimal Treg function in disease models [25].
HIF-1 $\alpha$ is also essential for normal B-cell development and self-tolerance in a chimeric HIF- $1 \alpha^{-/-} \Rightarrow \mathrm{Rag} 2^{-/-}$murine model [26].

In RA, HIF- $1 \alpha$ induces both angiogenesis and proinflammatory mechanisms [27], and in lupus nephritis larger amounts of HIF- $1 \alpha$ in both glomerular and tubulointerstitial areas evaluated by immunohistochemical staining have been observed [28].

Thus, there is significant evidence that the HIF- $1 \alpha$ pathway is important to several aspects of innate and adaptive immune function, differentiation, and cytokine expression. Herein we identify that the HIF1A Pro582Ser (rs11549465) $T$ 
allele and $C / T$ genotype were protective for pSS. Interestingly, in a HIF- $1 \alpha$ conditional knockout mouse model with dry-eye, the lacrimal glands exhibited increased acinar cell apoptosis and loss of glandular structures and polarities, and the blockage of HIF- $1 \alpha$ COX-2 axis enhanced inflammation [10]. This evidence suggests a potential protective effect of Pro568Ser HIF1A polymorphism in pSS.

HIF1A Pro582Ser (rs11549465) polymorphism is located in the 12th exon of the gene and confers a substitution of a proline to a serine in the 582-amino acid residue of the codified protein. This polymorphism fell in the oxygen-dependent degradation domain of HIF- $1 \alpha$ and in one of its transactivation domains suggesting its participation in HIF- $\alpha$ function [29]. Previously in vitro studies have showed that the carriers of these polymorphic variants confer greater stability and promote an elevated transcriptional activity of HIF- $1 \alpha$ when compared to the wild type, under normoxic and hypoxic conditions [30, 31].

These works point out the relevance and functionality of this polymorphism over the transcription of HIF- $1 \alpha$ target genes that are involved in pSS pathology.

Previous genetic studies in pSS rarely reported association of candidate genes with clinical features. For instance, a trend for association between genetic variation in SNP of SLC25A40 and PKN1 and fatigue has been reported [32]. Herein we observed that HIF1A Pro582Ser (rs11549465) $T$ allele and $C / T$ genotype protected against parotid enlargement among the pSS group.

On the other hand, AKNA is an AT-hook transcription factor, which upregulates the expression of costimulatory cell surface molecules, such as CD40 and CD154, on immune response cells [33]. These costimulatory molecules are critical for the triggering of the humoral response in B cells and priming of T lymphocytes [34]. In pSS patients, the concentration of CD154 in serum and the expression of CD154 in T CD4+ cells are significantly higher compared with healthy controls $[35,36]$. Interestingly, the overexpression of CD154 reported by Belkhir et al. [37] was not due to a difference in DNA methylation patterns. This points out the relevance of AKNA as transcription regulator of CD154 and CD40 in pSS pathology.

Herein we found that the $A K N A-1372 C>A$ (rs10817595) $A / A$ genotype under a recessive inheritance model was associated with an increased risk of pSS. In contrast, loss of AKNA in a murine model results in mice that die suddenly at 10 days of life with diffuse inflammatory lesions predominantly in the lungs, induced mainly by IL-1- $\beta$ and IFN- $\gamma$ and development of anti-DNA autoantibodies [38].

The polymorphism associated with higher risk of pSS in the present work $(-1372 C>A$, rs10817595) is located in the promoter region of $A K N A$ gene. This polymorphism has been proposed as an expression quantitative trait loci (eQTL), given that in peripheral blood mononuclear cells the $A$ allele promotes higher expression level of AKNA [14].

To date, the molecular mechanism involved in $H I F 1 A$ and $A K N A$ expression is still a matter of research. For instance, HIF1A can extend different and sometimes opposing roles and this is probably sustained by their unusual capacity to generate multiple transcripts and proteins. In addition, the apparent conflicting results in knockout-deletion mice models for both genes should be taken with caution regarding overreliance on these models in isolation.

Genome-wide association studies (GWAS) are an important tool for identifying disease susceptibility genes. The existing GWAS have not identified HIF1A and AKNA as genes associated with the disease $[4,39]$. Considering that the majority of these GWAS have been done in Caucasian or European populations, the results do not necessarily provide information for the Mexican population. In addition, the genetic risk varies across ethnicity and genetic heterogeneity is a well-recognized reason for failure to replicate genetic association findings. In fact a recent GWAS study among European and Asian SS patients showed striking differences between both populations, with high heterogeneity especially in the MHC and representative SNPs. Thus genetic associations with SS differ markedly by ancestry [40]. Moreover, it is known that GWAS have limitations to detect low frequency and small magnitude variants associated with the disease in question.

A possible limitation of this study is the fact that we observed Hardy-Weinberg disequilibrium (HWD) in the control group for AKNA -1372C>A (rs10817595) and HIF1A Pro582Ser (rs11549465) polymorphisms. It is highly unlikely that the HWD found was due to genotyping errors because in each assay we had previously genotyped positive controls, and we had $100 \%$ of concordance in each performed assay. Besides, taking into account the Bonferroni correction for multiple comparisons the $P$ values for these HWE tests were highly above the significance level. Furthermore, the allele frequencies observed for the polymorphism in which the HWE $P$ value was the lowest (AKNA rs10817595) are consistent with that reported in the 1000 genomes project for Mexicans ( $41 \%$ for the $A$ allele and 59\% for the $C$ allele) [41].

In addition as pSS is a disease that primary affects women between ages 30 and 50 and our controls were predominantly men and young, we adjusted our results by gender and age in order to minimize any possible influence that these variables could have over the associations found.

In conclusion, we identified HIF1A Pro582Ser (rs11549465) $T$ allele and $C / T$ genotype as well as $A K N A$ $-1372 C>A$ (rs10817595) $A / A$ genotype as susceptibility genetic factors of pSS, conferring the former a decreased and the latter an increased risk of pSS among Mexican mestizo population. Notwithstanding, as these individual polymorphisms only confer a small risk change, further studies in other populations are needed to validate our findings and to assess their participation in additive models with other known risk alleles as well as with their interaction with epigenetic and environmental factors. Therefore, research is warranted in order to shed some light on their functional implications across biological pathways in this disease.

\section{Disclosure}

This research did not receive any specific grant from funding agencies in the public, commercial, or not-for-profit sectors. 


\section{Conflicts of Interest}

The authors declare no conflicts of interest.

\section{References}

[1] M. Ramos-Casals and J. Font, "Primary Sjögren's syndrome: current and emergent aetiopathogenic concepts," Rheumatology, vol. 44, no. 11, pp. 1354-1367, 2005.

[2] J. M. Kramer, "Early events in Sjögren's Syndrome pathogenesis: the importance of innate immunity in disease initiation," Cytokine, vol. 67, no. 2, pp. 92-101, 2014.

[3] W. G. Feero, A. E. Guttmacher, and F. S. Collins, "Genomic medicine-an updated primer," The New England Journal of Medicine, vol. 362, no. 21, pp. 2001-2011, 2010.

[4] P. D. Burbelo, K. Ambatipudi, and I. Alevizos, "Genome-wide association studies in Sjögren's syndrome: what do the genes tell us about disease pathogenesis?" Autoimmunity Reviews, vol. 13, no. 7, pp. 756-761, 2014.

[5] G. L. Semenza, "HIF-1: mediator of physiological and pathophysiological responses to hypoxia," Journal of Applied Physiology, vol. 88, no. 4, pp. 1474-1480, 2000.

[6] J. Fernández-Torres, C. Hernández-Díaz, R. Espinosa-Morales et al., "Polymorphic variation of hypoxia inducible factor-1 a (HIF1A) gene might contribute to the development of knee osteoarthritis: a pilot study," BMC Musculoskeletal Disorders, vol. 16, no. 1, article 218, 2015.

[7] A. Palazon, A. W. Goldrath, V. Nizet, and R. S. Johnson, "HIF transcription factors, inflammation, and immunity," Immunity, vol. 41, no. 4, pp. 518-528, 2014.

[8] E. V. Dang, J. Barbi, H.-Y. Yang et al., "Control of TH17/Treg balance by hypoxia-inducible factor 1," Cell, vol. 146, no. 5, pp. 772-784, 2011.

[9] A. Sakai, Y. Sugawara, T. Kuroishi, T. Sasano, and S. Sugawara, "Identification of IL-18 and Th17 cells in salivary glands of patients with Sjögren's syndrome, and amplification of IL-17mediated secretion of inflammatory cytokines from salivary gland cells by IL-18," The Journal of Immunology, vol. 181, no. 4, pp. 2898-2906, 2008.

[10] Y. Seo, Y. W. Ji, S. M. Lee et al., "Activation of HIF-1 $\alpha$ (hypoxia inducible factor- $1 \alpha$ ) prevents dry eye-induced acinar cell death in the lacrimal gland," Cell Death \& Disease, vol. 5, no. 6, Article ID e1309, 2014.

[11] S. Hua and T. H. Dias, "Hypoxia-Inducible Factor (HIF) as a target for novel therapies in rheumatoid arthritis," Frontiers in Pharmacology, vol. 7, article 184, 2016.

[12] Z.-C. Yang and Y. Liu, "Hypoxia-inducible factor- $1 \alpha$ and autoimmune lupus, arthritis," Inflammation, vol. 39, no. 3, pp. 12681273, 2016.

[13] J. C. Sims-Mourtada, S. Bruce, M. R. McKeller et al., "The human AKNA gene expresses multiple transcripts and protein isoforms as a result of alternative promoter usage, splicing, and polyadenylation," DNA and Cell Biology, vol. 24, no. 5, pp. 325338, 2005.

[14] G. A. Martínez-Nava, K. Torres-Poveda, A. Lagunas-Martínez et al., "Cervical cancer-associated promoter polymorphism affects AKNA expression levels," Genes and Immunity, vol. 16, no. 1, pp. 43-53, 2015.

[15] C. Chen, C. Bartenhagen, M. Gombert et al., "Next-generationsequencing of recurrent childhood high hyperdiploid acute lymphoblastic leukemia reveals mutations typically associated with high risk patients," Leukemia Research, vol. 39, no. 9, pp. 990-1001, 2015.

[16] L. Mao, P. Yang, S. Hou, F. Li, and A. Kijlstra, "Label-free proteomics reveals decreased expression of CD18 and AKNA in Peripheral CD4+ T Cells from patients with Vogt-KoyanagiHarada syndrome," PLoS ONE, vol. 6, no. 1, Article ID e14616, 2011.

[17] N. Papic, C. I. Maxwell, D. A. Delker, S. Liu, B. S. E. Heale, and C. H. Hagedorn, "RNA-sequencing analysis of 5' capped RNAs identifies many new differentially expressed genes in acute hepatitis C virus infection," Viruses, vol. 4, no. 4, pp. 581612,2012

[18] C. Vitali, S. Bombardieri, R. Jonsson et al., "Classification criteria for Sjögren's syndrome: a revised version of the European criteria proposed by the American-European Consensus Group," Annals of the Rheumatic Diseases, vol. 61, no. 6, pp. 554$558,2002$.

[19] D. K. Lahiri and J. I. Numberger Jr., "A rapid non-enzymatic method for the preparation of HMW DNA from blood for RFLP studies," Nucleic Acids Research, vol. 19, article 5444, 1991.

[20] J.-H. Tao, J. Barbi, and F. Pan, "Hypoxia-inducible factors in $\mathrm{T}$ lymphocyte differentiation and function. A review in the theme: cellular responses to hypoxia," American Journal of Physiology-Cell Physiology, vol. 309, no. 9, pp. C580-C589, 2015.

[21] L. D'Ignazio, D. Bandarra, and S. Rocha, "NF- $\kappa$ B and HIF crosstalk in immune responses," FEBS Journal, vol. 283, no. 3, pp. 413-424, 2016.

[22] S. A. Gerber and J. S. Pober, "IFN- $\alpha$ induces transcription of hypoxia-inducible factor- $1 \alpha$ to inhibit proliferation of human endothelial cells," Journal of Immunology, vol. 181, no. 2, pp. 1052-1062, 2008.

[23] F. Hu, H. Liu, L. Xu et al., "Hypoxia-inducible factor- $1 \alpha$ perpetuates synovial fibroblast interactions with T cells and B cells in rheumatoid arthritis," European Journal of Immunology, vol. 46, no. 3, pp. 742-751, 2016.

[24] A. Nezos, F. Gravani, A. Tassidou et al., “Type I and II interferon signatures in Sjogren's syndrome pathogenesis: contributions in distinct clinical phenotypes and Sjogren's related lymphomagenesis," Journal of Autoimmunity, vol. 63, pp. 47-58, 2015.

[25] E. T. Clambey, E. N. McNamee, J. A. Westrich et al., "Hypoxiainducible factor-1 alpha-dependent induction of FoxP3 drives regulatory $\mathrm{T}$-cell abundance and function during inflammatory hypoxia of the mucosa," Proceedings of the National Academy of Sciences of the United States of America, vol. 109, no. 41, pp. E2784-E2793, 2012.

[26] H. Kojima, H. Gu, S. Nomura et al., "Abnormal B lymphocyte development and autoimmunity in hypoxia-inducible factor $1 \alpha$ deficient chimeric mice," Proceedings of the National Academy of Sciences of the United States of America, vol. 99, no. 4, pp. 2170$2174,2002$.

[27] W. Gao, J. McCormick, M. Connolly et al., "Hypoxia and STAT3 signalling interactions regulate pro-inflammatory pathways in rheumatoid arthritis," Annals of the Rheumatic Diseases, vol. 74, no. 6, pp. 1275-1283, 2015.

[28] W. Deng, Y. Ren, X. Feng et al., "Hypoxia inducible factor-1 alpha promotes mesangial cell proliferation in lupus nephritis," American Journal of Nephrology, vol. 40, no. 6, pp. 507-515, 2014.

[29] S. C. Clifford, D. Astuti, L. Hooper, P. H. Maxwell, P. J. Ratcliffe, and E. R. Maher, "The pVHL-associated SCF ubiquitin ligase complex: molecular genetic analysis of elongin $\mathrm{B}$ and $\mathrm{C}$, Rbxl 
and HIF-1 $\alpha$ in renal cell carcinoma," Oncogene, vol. 20, no. 36, pp. 5067-5074, 2001.

[30] K. Tanimoto, K. Yoshiga, H. Eguchi et al., "Hypoxia-inducible factor- $1 \alpha$ polymorphisms associated with enhanced transactivation capacity, implying clinical significance," Carcinogenesis, vol. 24, no. 11, pp. 1779-1783, 2003.

[31] J. M. Hong, T.-H. Kim, S.-C. Chae et al., "Association study of hypoxia inducible factor $1 \alpha$ (HIF $1 \alpha)$ with osteonecrosis of femoral head in a Korean population," Osteoarthritis and Cartilage, vol. 15, no. 6, pp. 688-694, 2007.

[32] K. B. Norheim, S. Le Hellard, G. Nordmark et al., "A possible genetic association with chronic fatigue in primary Sjögren's syndrome: a candidate gene study," Rheumatology International, vol. 34, no. 2, pp. 191-197, 2014.

[33] A. Siddiqa, J. C. Sims-Mourtada, L. Guzman-Rojas et al., "Regulation of CD40 and CD40 ligand by the AT-hook transcription factor AKNA," Nature, vol. 410, no. 6826, pp. 383-387, 2001.

[34] M. F. Mackey, J. R. Gunn, C. Maliszewsky, H. Kikutani, R. J. Noelle, and R. J. Barth Jr., "Dendritic cells require maturation via CD40 to generate protective antitumor immunity," The Journal of Immunology, vol. 161, no. 5, pp. 2094-2098, 1998.

[35] A. L. Peters, L. L. Stunz, and G. A. Bishop, "CD40 and autoimmunity: the dark side of a great activator," Seminars in Immunology, vol. 21, no. 5, pp. 293-300, 2009.

[36] A. Goules, A. G. Tzioufas, M. N. Manousakis, K. A. Kirou, M. K. Crow, and J. G. Routsias, "Elevated levels of soluble CD40 ligand (sCD40L) in serum of patients with systemic autoimmune diseases," Journal of Autoimmunity, vol. 26, no. 3, pp. 165-171, 2006.

[37] R. Belkhir, N. Gestermann, M. Koutero et al., "Upregulation of membrane-bound CD40L on CD4+ T cells in women with primary Sjögren's syndrome," Scandinavian Journal of Immunology, vol. 79, no. 1, pp. 37-42, 2014.

[38] W. Ma, B. Ortiz-Quintero, R. Rangel et al., "Coordinate activation of inflammatory gene networks, alveolar destruction and neonatal death in AKNA deficient mice," Cell Research, vol. 21, no. 11, pp. 1564-1577, 2011.

[39] I. Song, H. Chen, Y. Lin et al., "Identification of susceptibility gene associated with female primary Sjögren's syndrome in Han Chinese by genome-wide association study," Human Genetics, vol. 135, no. 11, pp. 1287-1294, 2016.

[40] K. E. Taylor, Q. Wong, D. M. Levine et al., "Genome-wide association analysis reveals genetic heterogeneity of Sjögren's syndrome according to ancestry," Arthritis \& Rheumatology, 2017.

[41] G. R. Abecasis, A. Auton, L. D. Brooks et al., "An integrated map of genetic variation from 1,092 human genomes," Nature, vol. 491, pp. 56-65, 2012. 


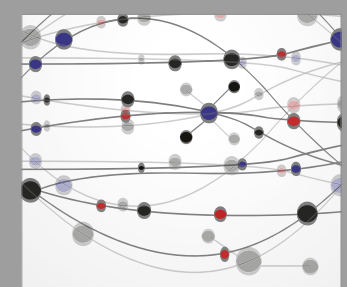

The Scientific World Journal
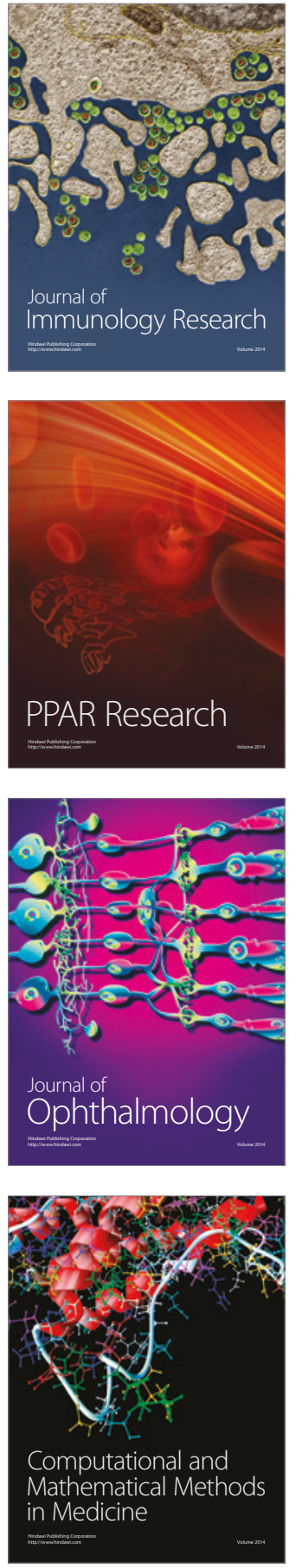

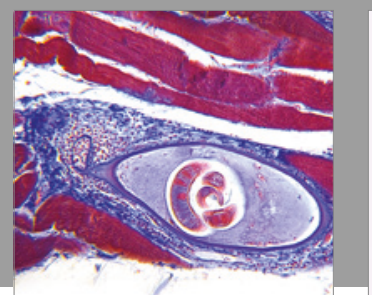

Gastroenterology Research and Practice
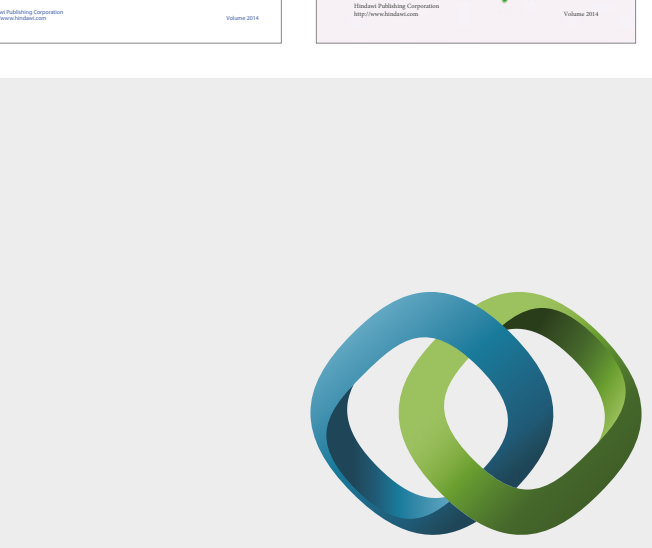

\section{Hindawi}

Submit your manuscripts at

https://www.hindawi.com
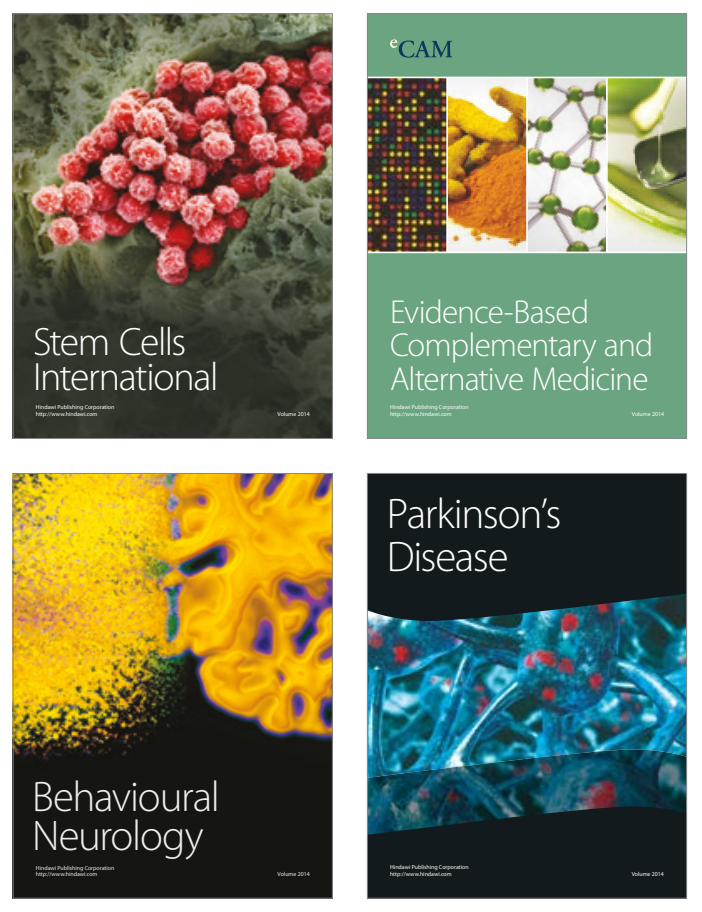
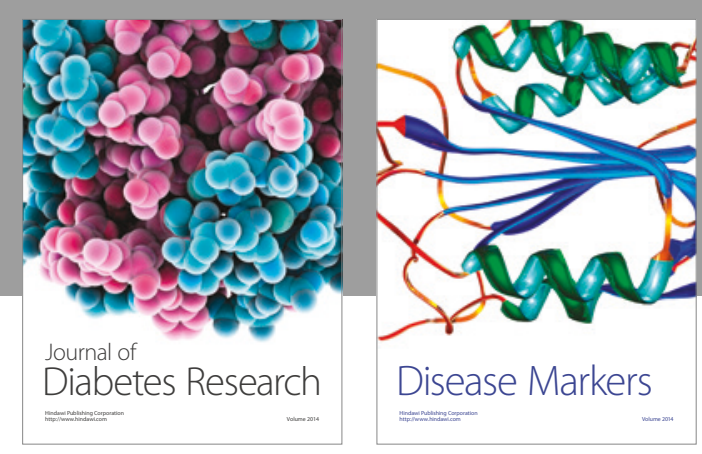

Disease Markers
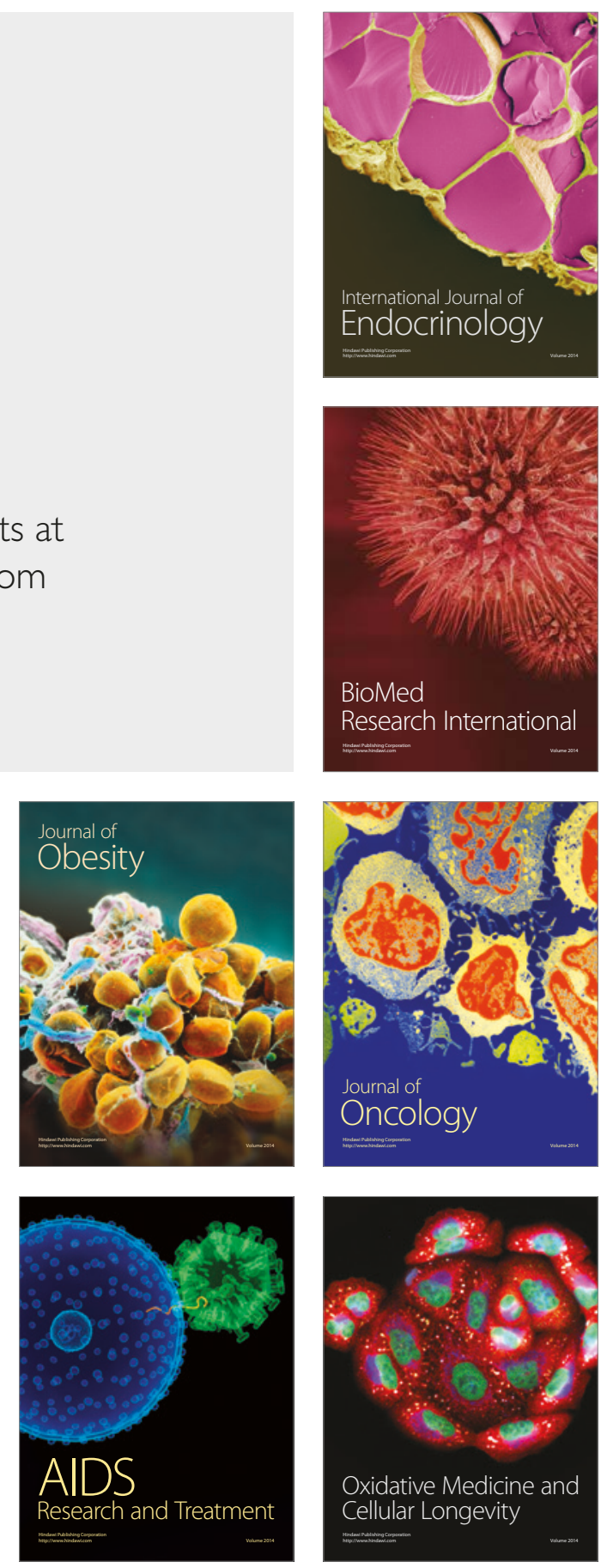